\title{
The use of Bayesian methodology in the development and validation of a tiered assessment approach towards prediction of rat acute oral toxicity
}

\author{
James W. Firman ${ }^{1}$ (D) Mark T. D. Cronin ${ }^{1} \cdot$ Philip H. Rowe ${ }^{1} \cdot$ Elizaveta Semenova $^{2} \cdot$ John E. Doe $^{1}$
}

Received: 17 September 2021 / Accepted: 9 December 2021 / Published online: 16 January 2022

(c) The Author(s) 2022

\begin{abstract}
There exists consensus that the traditional means by which safety of chemicals is assessed-namely through reliance upon apical outcomes obtained following in vivo testing-is increasingly unfit for purpose. Whilst efforts in development of suitable alternatives continue, few have achieved levels of robustness required for regulatory acceptance. An array of "new approach methodologies" (NAM) for determining toxic effect, spanning in vitro and in silico spheres, have by now emerged. It has been suggested, intuitively, that combining data obtained from across these sources might serve to enhance overall confidence in derived judgment. This concept may be formalised in the "tiered assessment" approach, whereby evidence gathered through a sequential NAM testing strategy is exploited so to infer the properties of a compound of interest. Our intention has been to provide an illustration of how such a scheme might be developed and applied within a practical setting-adopting for this purpose the endpoint of rat acute oral lethality. Bayesian statistical inference is drawn upon to enable quantification of degree of confidence that a substance might ultimately belong to one of five LD50-associated toxicity categories. Informing this is evidence acquired both from existing in silico and in vitro resources, alongside a purposely-constructed random forest model and structural alert set. Results indicate that the combination of in silico methodologies provides moderately conservative estimations of hazard, conducive for application in safety assessment, and for which levels of certainty are defined. Accordingly, scope for potential extension of approach to further toxicological endpoints is demonstrated.
\end{abstract}

Keywords New approach methodologies $\cdot$ Bayesian inference $\cdot$ Tiered assessment $\cdot$ Regulatory toxicology $\cdot$ Acute toxicity · In silico toxicology

\section{Introduction}

The publication of "Toxicity Testing in the 21st Century" set an expectation that the manner in which chemical safety is assessed with regards to human health would soon radically change (Krewski et al. 2010). Since that time, many initiatives have emerged based upon adoption of novel methodologies (broadly termed New Approach Methodologies, or NAM) (Dal Negro et al. 2018). However, the bulk of regulatory decision-making, a fifth of the way through the

James W. Firman

j.w.firman@1jmu.ac.uk

1 School of Pharmacy and Biomolecular Sciences, Liverpool John Moores University, Liverpool, UK

2 Department of Mathematics, Imperial College London, London, UK twenty-first century, remains grounded in traditional laboratory animal-based techniques developed in the third quarter of the 20th. Regulators in the 1970s would not have been content with making use of methods devised in the 1920s, so why should we in the 2020 s continue to rely on strategies similarly developed half-a-century ago?

Many NAM have been developed, both in vitro and in silico, with the aim of assessing biological effect, thereby enabling extrapolation to the in vivo sphere. The former includes a plethora of biomaterial-based systems incorporating either whole cells or their components (Anadón et al. 2014), whereas the latter encompasses techniques spanning quantitative structure-activity relationship (QSAR) modelling, machine learning and the creation of structural alerts (Madden et al. 2020). The rationalisation of NAM findings may be underpinned by concepts such as the Adverse Outcome Pathway (AOP) - which charts mode and mechanism of toxic action through stages including molecular initiating 
and key events (typically cellular), to ultimate adverse outcome at the organ and organism-level (Edwards et al. 2016; Tollefsen et al. 2014; Vinken 2013). Despite such progress, regulatory adoption of new approaches has remained slow (Knight et al. 2021). Whilst many validation programmes have been attempted, a lack of confidence in the general applicability of NAM continues to exist (Parish et al. 2020). We postulate that there are at least two fundamental reasons behind the persistence of this sense of scepticism: potential flaws within the methodologies themselves, and concerns regarding the lack of a means towards validation. Both factors may contribute towards lower acceptability for regulatory use (Mahony et al. 2020).

The twin factors of flawed methodology and inappropriate validation are interlinked. Initially, in the search for techniques not involving the use of laboratory animals, individual approaches were assessed directly for their ability to reproduce the results of the in vivo protocol which they were intended to replace (Piersma et al. 2018). Outcomes of single tests were compared against those of animal studies, to gauge the number "correct" answers matched. This was often performed using a sensitivity/specificity paradigm based upon a simplistic conception of "wrong and right answers", in turn leading to concerns over the occurrence of false positive and false negative results. There are issues with this approach. Firstly, given the level of complexity entailed, it is unrealistic to expect that a single test might reliably reproduce the results of an animal study: a combination of sources shall more likely produce success (Piersma et al. 2014). Secondly, there is very little in toxicology which is truly binary: a continuum is usually present, with "positive" and "negative" judgments dictated by the side of a line on which the answer lies.

The concept of compounding data derived from an assortment of in silico, in vitro and in vivo sources, to produce a tiered assessment of toxic potential, has in recent years been advanced as a means of addressing each of these broad issues (Andersen et al. 2019; Thomas et al. 2013, 2019). Such an approach may not merely allow for the enhancement of confidence in prediction relative to isolated NAM (through intensifying weight-of-evidence), but may further be adapted to facilitate quantitative expression of certaintyextending resolution beyond the simple binary call. For this to be realised, a statistical methodology must be adopted through which the outputs of the various approaches can be integrated to produce updated judgments. Bayesian inference represents a powerful technique for achieving this, permitting as it does the generation of probabilistic distributions which may be related to severity of toxicity (Lazic and Williams 2021). Its application within predictive toxicology constitutes an emerging field of interest, and as such it has been drawn upon in recent studies aimed towards development of models describing endpoints spanning skin sensitivity (Reynolds et al. 2019), drug-induced liver injury (Semenova et al. 2020; Williams et al. 2020) and cardiotoxicity (Felli and Leishman 2020).

This paper describes a route towards the development and application of such a tiered approach - adopting, for illustrative purposes, the assessment of acute oral lethality within the rat. Bayesian methodology is employed to compute distributions relating the probability that a given substance might belong to one of five categories, each corresponding to a defined LD50 range mirroring those adopted within European Union Classification, Labelling and Packaging (CLP) regulation. Three tiers are incorporated, over which a variety of in silico and in vitro methodologies are exploited. In Bayesian terms, the outputs from the previous tier are adopted as the "prior" to inform that which follows. Analysis of predictive quality following introduction of each tier enables the certainty of its outcomes to be determined, in turn allowing for the contribution of the constituent techniques to be discerned. Alongside the sourcing of data from existing QSAR schemes, the training of novel machine learning algorithms and structural alert sets are reported. In addition to validating the tiered approach developed, our intention is to demonstrate how the output from such a system may be appropriately evaluated. Through this, it should be possible to ascertain whether or not the strategy is ultimately successful in its aims of improving, in principle, the acceptability of predictions derived from NAM.

\section{Materials and methods}

\section{Sourcing of rat acute oral toxicity data}

From the publication of Gadaleta et al. (2019), data describing acute oral toxicity towards rats within an inventory of organic substances were acquired. These data are themselves drawn from a yet wider selection collated through efforts of NICEATM and US EPA (https://ntp.niehs.nih.gov/go/ tox-models; accessed 1-5-2020)—with final LD50 quantities relating to compounds possessing greater than three distinct point estimates being derived in accordance with methodology outlined by Nelms et al. (2020). Contained within the Gadaleta et al. dataset were records describing 11,363 substances, of which 8448 contained accompanying values relating experimental toxicity (LD50, expressed as $\left.\mathrm{mg} / \mathrm{kg}_{\mathrm{bw}}\right)$. Through removal both of duplicate entries and of those possessing undefined chemical structure (including mixtures and polymers), a final working set consisting of 8186 distinct organic molecules was formulated. Existing SMILES (www.daylight.com) (Weininger 1988) strings were retained, whilst chemical names were related to supplied CASRN either through use of the US EPA CompTox Chemicals Dashboard (https://comptox.epa.gov/dashb 
oard; accessed 1-5-2020) (Williams et al. 2017) or through PubChem (https://pubchem.ncbi.nlm.nih.gov/; accessed 1-5-2020). For purposes of model development, LD50 estimates were transformed directly into their $\log \mathrm{mmol} / \mathrm{kg}_{\mathrm{bw}}$ equivalents. Data are presented in full within Supplementary Table 1.

\section{Assignment of acute toxicity category to compounds}

In accordance with the scheme outlined within Table 1, chemicals were each assigned a numerical acute oral toxicity category (1-5) derived directly from acute oral LD50. This rubric is itself adapted from that specified within European Council Regulation No. 1272/2008 (European Union 2008) - a minor amendment being the appending of a "Category 5", covering compounds holding LD50 greater than $2000 \mathrm{mg} / \mathrm{kg}_{\mathrm{bw}}$.

\section{Derivation of Cramer scheme classification}

The Cramer classification scheme assigns molecules into one of three "threshold of toxicological concern (TTC)" classes, describing broadly and conservatively their level of hazard towards human health under a repeat-exposure scenario (EFSA Scientific Committee 2019; Cramer et al. 1978). It is constituted as a decision tree, with presence or absence of characteristic chemical structural features determining the final placement of a compound. Class I corresponds to substances of least apparent toxic concern, Class II to substances of intermediate concern and Class III to substances of greatest concern. Cramer classifications for each compound within the working set were sourced though use of the "Cramer rules, with extensions" decision tree (Patlewicz et al. 2008), as implemented within ToxTree software (v. 3.1.0; EU Joint Research Centre and IDEAconsult; http:// toxtree.sourceforge.net/). Classes were definitively assigned to 8180 of the 8186 chemicals within.

It was necessary that probability distributions were derived describing the relationship associating assigned classification with experimentally derived acute toxicity category. Raw distributions were initially acquired, based

Table 1 Overview of scheme through which acute toxicity category is assigned from oral LD50 upon a simple count of the occurrence of toxicity categories amongst those compounds sharing a class. By way of illustration, amongst the 7097 substances assigned Class III, 219 (3.1\%) fell into Category 1 based upon their in vivo LD50, 629 (8.9\%) Category 2, 1385 (19.5\%) Category 3, 3003 (42.3\%) Category 4 and 1861 (26.2\%) Category 5. However, adjustments were required on account of the marked imbalance present within the spread of experimental toxicity across the 8136 compounds forming the dataset. To achieve this, these raw counts were scaled in proportion to the occurrence of each category within experimental data (listed within Table 4, column "Distribution"). Given that $40.6 \%$ of compounds fell into Category 4 and $2.7 \%$ into Category 1 , raw percentages for each were divided respectively by 0.406 and 0.027 . Applying this to all categories, final adjusted distributions were acquired.

\section{Prediction of acute rat oral LD50 through use of US EPA Toxicity Estimation Tool}

Contained within the US EPA Toxicity Estimation Tool (TEST) (v. 4.2.1; US EPA; https://www.epa.gov/chemicalresearch/toxicity-estimation-software-tool-test) is a variety of QSAR models relating directly to assignment of acute oral LD50 within the rat. Through use of the "hierarchical clustering" method, LD50 predictions could be acquired for 7479 of the 8186 screened substances. A detailed description of the methodology underlying this QSAR may be found within Martin et al. (2008).

\section{Development of random forest model for estimation of acute rat oral LD50}

Relationship between chemical structure, calculated molecular physicochemical properties and corresponding acute oral LD50 was further modelled through the training of a random forest algorithm. Employed as input variables were a combination of molecular fingerprint fragments and physicochemical descriptors. Screening of compounds for their PubChem substructure fingerprint (ftp://ftp.ncbi.nlm.nih. gov/pubchem/specifications/pubchem_fingerprints.txt) was performed within KNIME software (v. 4.3.1; www.knime. com) (Berthold et al. 2008), through use of the RDKit (v. 2020.03.6; www.rdkit.org) (Landrum 2006) Fingerprint node. Fragments present in fewer than $5 \%$ or in greater than $95 \%$ of compounds were excluded from consideration, whilst all others (totalling 300) were retained. An assortment of 1D and 2D Molecular physicochemical descriptors were sourced from Molecular Operating Environment (MOE) software (v. 2018.01; Chemical Computing Group; https://www.chemcomp.com/) (Molecular Operating Environment 2018). Following removal of parameters unsuitable for modelling, a sum of 184 descriptors remained. Pooling 
with the aforementioned fingerprint fragments produced a set of 484 variables, characterising the complete collection of 8186 compounds. This full parameter set is available from the authors upon request. Employing $\log \mathrm{mmol} / \mathrm{kg}_{\mathrm{bw}} \mathrm{LD} 50$ as the modelled quantity, a random forest consisting of 500 trees was constructed within R (v. 4.0.1; R Foundation; https://www.r-project.org/) (R Core Team 2021), through use of RStudio (https://www.rstudio.com/) (RStudio Team 2020) and the randomForest package (Liaw and Wiener 2002). Applying ten-fold cross-validation, predictions for all modelled compounds were obtained.

\section{Compilation of molecular structural alerts associated with elevated acute oral toxicity}

A series of tailored chemical structural alerts were developed, to assess the broader utility of the approach in enhancing the correct identification of potentially hazardous compounds-notably, those for which corresponding toxicity in vivo is liable to be understated through alternative methods such as QSAR or in vitro cytotoxicity screening. Such groups were identified through manual analysis of output from TEST hierarchical clustering, random forest and in vitro systems - and further rationalised through application of expert judgment. Broadly, the highlighted structures could be considered to fall into one of two varieties: those integral within mediating a mechanism of toxic action not reliably recapitulated within simple $2 \mathrm{D}$ cell-based assay systems (such as neurotoxicity or anticoagulant effect), or those representative of specific compound classes (typically complex natural products) not adequately modelled through global QSAR.

For those alerts which were present within a minimum of ten compounds, distributions relating the probability of a substance falling within a given acute toxicity category were determined through a method similar to that described within 2.3 (with raw counts scaled in proportion to the prevalence of each category within the experimental data). In several instances, the fragment forming the alert occurs within fewer than ten compounds across the dataset.
Distributions in such cases are manually estimated, with probability of falling within Category 1 assigned at $90 \%$, Category 4 at $10 \%$ and the remaining categories each $0 \%$.

\section{Acquisition of in vitro cytotoxicity data}

Data relating to cytotoxicity in vitro were gathered initially from a variety of sources, including the publications of Clothier et al. (2008), Prieto et al. (2013) and KinsnerOvaskainen et al. (2013). However, owing to the very limited overlap apparent between the compounds present within these studies and those comprising the LD50 dataset (data not shown), their use was deemed impractical.

Cell viability assays present across the Tox Cast (Richard et al. 2016) and Tox 21 (Tice et al. 2013) platforms (accessed through US EPA CompTox Chemicals Dashboard) were subsequently examined for their suitability, in terms both of raw coverage and quality of correlation of AC50 with acute in vivo LD50. Assay “TOX21_RT_HEK293_GLO_16HR_ VIABILITY" was found to be optimal in this regard (data not shown), covering a total of 543 substances. In brief, the technique assessed viability of HEK-293 (human embryonic kidney) cells following $16 \mathrm{~h}$ treatment with substance of interest, though a bioluminometric protocol.

\section{Overview of tiered approach}

The aforementioned methodologies were portioned into one of three "assessment tiers", each representing distinct approaches towards hazard prediction (Cramer classification, in silico and in vitro_-as outlined within Table 2). Further reference is made within to a hypothetical "Tier 3", in which it is envisioned that a refined in vivo protocol, such as that exemplified by OECD Test Guideline 425 (OECD 2008), would be selectively initiated to address uncertainties existing in category assignment towards specific compounds (an idea largely beyond the scope of this study, yet considered further within Discussion). The concordance of predicted and experimentally determined acute toxicity categories were assessed following application of each tier, to validate

Table 2 Overview of assessment tiers, outlining the composition of each in relation to methodologies incorporated

\begin{tabular}{lllc}
\hline Assessment tier & Approach overview & Methodologies incorporated & Category assignment \\
\hline 0 & Cramer classification & Cramer scheme (with extensions) & $\begin{array}{c}\text { Classification-category } \\
\text { probability distribution } \\
\text { (Table 5) }\end{array}$ \\
& & & Bayesian model \\
1 & In silico & EPA TEST LD50 & \\
& & Random forest LD50 & Bayesian model \\
3 (hypothetical) & In vitro & Structural alerts & Experimental outcome
\end{tabular}


the contribution of each towards improving (or otherwise) the quality of forecast - both in terms of the proportion of compounds correctly assigned, and of those either under- or overpredicted. Details of the Bayesian model employed in attributing categories within Tier 1 and Tier 2 are provided within section "Bayesian analysis".

\section{Application of tiered approach}

The tiered predictive approach was subsequently applied to a representative selection of 50 compounds from out of the 8186 forming the working dataset. On account of the inherent imbalance present within the distribution of toxicity across the unfiltered set, it was necessary to ensure that these representatives were drawn in equivalent numbers from each of the five acute toxicity categories. To meet criteria for inclusion, substances had to have received a valid Cramer classification, LD50 assignments through both EPA TEST and random forest techniques, and data from the "TOX21_ RT_HEK293_GLO_16HR_VIABILITY” assay. Presence of a structural alert was, however, not a requirement.

A total of 496 molecules were found to meet the above stipulations. Ten each were drawn from Categories 3, 4 and 5. Since only six eligible substances were observed to fall into Category 1, an additional four were drawn from amongst those in Category 2 to account for shortfall. The composition of this selection may be found within Supplementary Table 2.

\section{Bayesian analysis}

Bayesian predictive models were constructed based upon proportional odds logistic regression (POLR). The outcome variable $y_{i}$ is represented by ordered categorical data, i.e. compound's acute toxicity category. For each compound $i$ the model calculates the underlying continuous severity $\eta_{i}$ based on available predictors $X_{i}$ using a set of regression coefficients $\beta_{i}$. A set of cut-points $c_{1}, \ldots, c_{4}$ subdivides the continuous severity to define boundaries between five discrete ordered categories. Regression coefficients and cutpoints are inferred from data. Tier 1 model incorporates the distribution emerging from Tier 0 , updating with outputs acquired from EPA TEST, random forest and (as applicable) from matched structural alerts. Subsequently, this is brought forward to Tier 2-whereby in vitro data is integrated to provide the ultimate posterior.

The Bayesian approach to model fitting requires specification of a likelihood and priors for each parameter. We adopt the priors from Williams et al. (2020), i.e. the regression coefficients are described by a Laplace distribution with mean $\mu$ and standard deviation $\sigma ; \mu$ and $\sigma$ are the hyperparameters of the model and are also estimated by the model. The cut-points are assigned weakly informative normal priors with mean 0 and standard deviation 20 . We used RStan interface (v. 2.21.2; http://mc-stan.org/) (Stan Development Team 2020) of the Stan modelling language (Carpenter et al. 2017). Predictions were derived from the samples obtained from four chains, 10,000 iterations each of the No-U-Turn sampling algorithm.

\section{Category attribution (exclusionary method)}

Assignment of predicted acute toxicity category was performed based upon categorical probability distributions obtained following application of each respective tier. In the instance of Tier 0 , this was the scaled probability distributions derived from Cramer classification. Bayesian models drawn from data presented within Tiers 1 and 2 produced corresponding outputs which were treated identically. For a given compound, all categories for which probability of belonging fell beneath a specified threshold (be it $10 \%$ or $5 \%$ ) were excluded from consideration (termed "exclusionary method"). Compounds were then attributed to the remaining category indicating the greatest hazard. This tended to produce conservative allocations, which would be preferable for purposes of risk assessment. By way of illustration, consider compounds holding distributions outlined within Table 3: applying a $10 \%$ threshold to Compound A leads to the exclusion of all with the exception of Categories 2 and 3. It is Category 2 , representing as it does greatest toxicity, which is assigned. Optional lowering of the threshold to 5\%
Table 3 Category-probability distributions relating to three hypothetical compounds-A, $\mathrm{B}$ and $\mathrm{C}$

\begin{tabular}{|c|c|c|c|c|c|c|c|}
\hline & \multicolumn{5}{|c|}{ Scaled probability distribution $(\%)$} & \multirow{2}{*}{\multicolumn{2}{|c|}{$\begin{array}{l}\begin{array}{l}\text { Category } \\
\text { assignment }\end{array} \\
\text { Threshold (\%) }\end{array}$}} \\
\hline & \multicolumn{5}{|c|}{ Acute toxicity category } & & \\
\hline & 1 & 2 & 3 & 4 & 5 & 5.0 & 10.0 \\
\hline Compound A & 8.0 & 55.0 & 30.0 & 4.0 & 3.0 & 1 & 2 \\
\hline Compound B & 0.0 & 5.0 & 15.0 & 50.0 & 30.0 & 2 & 3 \\
\hline Compound $\mathrm{C}$ & 0.0 & 0.0 & 1.0 & 9.0 & 90.0 & 4 & 5 \\
\hline
\end{tabular}

Acute toxicity categories, assigned following application of exclusionary thresholds $5.0 \%$ and $10.0 \%$, are listed 
expands the remit to incorporate Category 1 -which is accordingly granted. Compounds $\mathrm{B}$ and $\mathrm{C}$ provide further example of the approach in action.

\section{Results}

\section{Overview of data landscape}

Application of the acute toxicity categorisation scheme described within section "Assignment of acute toxicity category to compounds" to the collection of $8136 \mathrm{com}$ pounds possessing rat acute oral LD50 data produced the distribution outlined through Table 4. As is apparent, greater than $70 \%$ of substances possessed toxicity sufficient to place them within the two categories of least concern (4 and 5). By contrast, only approximately $10 \%$ fell within those representing the greatest hazard (1 and 2) - notable being neurotoxicants of the organophosphate and carbamate classes, vitamin $\mathrm{K}$-antagonist anticoagulants and various natural products (such as strychnine and saxitoxin) representative of wider toxin families.

It is informative to note that, although the in vivo experimental LD50 values described above are presented as point estimates, they are in fact the medians of a probability distribution. LD50 is usually determined through use of a probit calculation derived from lethality dose-response, with the median taken as a final value. In a prior analysis of this dataset (Kleinstreuer et al. 2018 ), it was reported that $28 \%$ of member chemicals possessed greater than two values corresponding to point estimates of acute oral LD50. This enabled evaluation of the variation in LD50 which ideally must be considered when framing concordance between the experimental LD50 and results derived from the tiered approach. It was determined that the $95 \%$ confidence limit for LD50 values was $\pm 0.31 \log _{10}(\mathrm{mg} / \mathrm{kg})$. It is thus illuminating to compare the calculated $95 \%$ confidence limits for the LD50 values, which is equivalent to a probability range of $0.62 \log _{10}(\mathrm{mg} / \mathrm{kg})$, with the ranges for categories $2-4$ which have both upper and lower limits. The limits for the classification categories are themselves 0.8 or $1 \log _{10}$ $(\mathrm{mg} / \mathrm{kg})$, and so it is likely that some chemicals with a single value would fall into a different category were the study to be repeated. Confusion matrices (Supplementary Table 3) were constructed to assess the impact of adding or subtracting $0.31 \log _{10}(\mathrm{mg} / \mathrm{kg})$ to the experimental value for the balanced subset of 50 LD50 values used to evaluate the tiered approach. It can be seen that $32 \%$ of all chemicals changed category when the confidence limit was added, whereas $24 \%$ switched when the limit was instead subtracted.
Table 4 Distribution of compounds in accordance with acute toxicity category occupied - expressed in terms both of raw quantity and of percentage of total

\begin{tabular}{llll}
\hline $\begin{array}{l}\text { Acute tox. } \\
\text { category }\end{array}$ & $\begin{array}{l}\text { LD50 range } \\
\left(\mathrm{mg} / \mathrm{kg}_{\mathrm{bw}}\right)\end{array}$ & $\begin{array}{l}\text { Number of } \\
\text { compounds }\end{array}$ & Distribution (\%) \\
\hline 1 & $<5$ & 219 & 2.7 \\
2 & $5-49$ & 638 & 7.8 \\
3 & $50-299$ & 1452 & 17.7 \\
4 & $300-1999$ & 3326 & 40.6 \\
5 & $\geq 2000$ & 2551 & 31.2 \\
\hline
\end{tabular}

\section{Cramer classification}

Scaled probability distributions related to Cramer classification are displayed within Table 5. As may be seen, the great majority of compounds were assigned Class III-that representing highest concern with respect to repeat-dose toxicity. Across those compounds falling within Classes I and II, clear trends are observed highlighting a similar, transferable association with reduced acute toxicity. Substances matching either call display steadily increasing probabilities of belonging to higher, more inert categories. This should be contrasted with Class III, for which the corresponding distribution is essentially flat.

\section{EPA test}

Predicted acute oral LD50 values (covering 7479 compounds) sourced using the US EPA TEST hierarchical clustering model were found to, in general, correlate well with experimental quantities ( $r^{2}$ of 0.739). Relationship is depicted graphically within Fig. 1.

\section{Random forest}

The performance of the random forest model in the prediction of LD50 was, overall, inferior to that of EPA TEST $\left(r^{2}\right.$ of 0.602). Relationship is depicted graphically within Fig. 2.

\section{Structural alerts}

A total of thirteen structural alerts were formulated, highlighting molecular fragments represented notably within compounds prevalent across acute toxicity categories 1 and 2. Dependent upon the absolute frequency of their occurrence within the dataset, category-probability distributions for each were either calculated or estimated. Details relating to those five features present with sufficient coverage so that their distributions were calculated may be found within Table 6.

Eight further alerts were identified-representative of those classes associated with high oral toxicity, yet present 
Table 5 Scaled categoryprobability distributions derived from Cramer classification

\begin{tabular}{|c|c|c|c|c|c|c|}
\hline & \multicolumn{5}{|c|}{ Scaled probability distribution (\%) } & \multirow{3}{*}{$\begin{array}{l}\text { Dataset } \\
\text { coverage } \\
(\%)\end{array}$} \\
\hline & \multicolumn{5}{|c|}{ Acute toxicity category } & \\
\hline & 1 & 2 & 3 & 4 & 5 & \\
\hline \multicolumn{7}{|c|}{ Cramer classification } \\
\hline I & 0.0 & 2.6 & 10.2 & 22.3 & 64.8 & 11.4 \\
\hline II & 0.0 & 7.6 & 13.3 & 25.2 & 53.8 & 1.9 \\
\hline III & 21.9 & 21.6 & 20.9 & 19.7 & 16.0 & 86.7 \\
\hline
\end{tabular}

only sparsely within the working dataset (fewer than ten compounds). Six of these described the core structural features of complex natural product families: aflatoxins, ochratoxins, saxitoxins, strychnine, trichothecenes and vitamins D. The remaining two corresponded, respectively to synthetic bromethalin neurotoxicants and indandione anticoagulants. Expanded description of all is presented within Supplementary Table 4.

\section{Cytotoxicity in vitro}

Of the variety of ToxCast cell viability assays examined, none were found to provide meaningful concordance with experimental acute oral LD50 (as noted within Section "Acquisition of in vitro cytotoxicity data"). Across the 50 compounds forming the balanced representative set, the settled-upon assay (TOX21_RT_HEK293_GLO_16HR_ VIABILITY) produced a correlation with $r^{2}$ merely 0.0877 . Figure 3 depicts this relationship graphically.

\section{Tiered approach}

Each of the 50 compounds selected to form a balanced, representative sample of the larger dataset (refer to Supplementary Table 2) were passed through the series of assessment

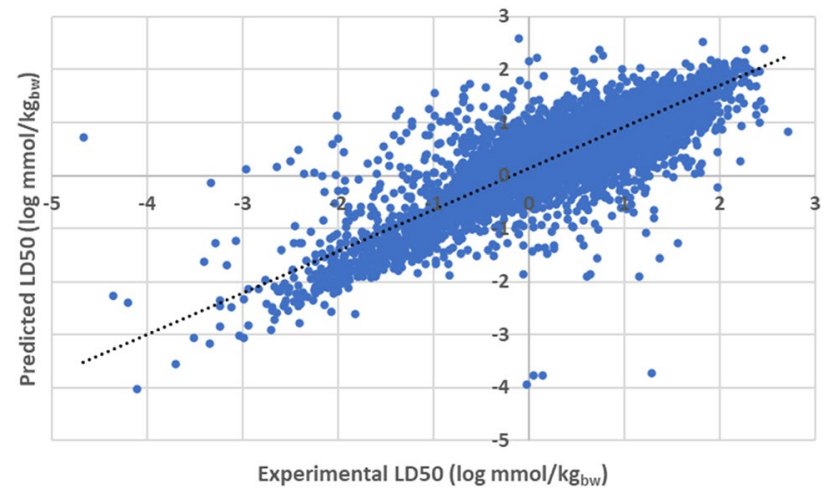

Fig. 1 Plot outlining correlation between acute oral LD50 as predicted through EPA TEST hierarchical clustering model, and that determined experimentally $\left(r^{2}=0.739\right)$ tiers outlined within section "Overview of tiered approach". Chosen primarily on account of the range of toxicity spanned, the sample cohort furthermore exhibited diversity with respect to chemical space and functional use-from comparatively simple molecules such as 4-tert-butyltoluene and 2-methylbutanal to complex natural products emetine and digitoxin. Examples of pharmaceuticals, endogenous biomolecules, synthetic intermediates and pesticides were each present.

\section{Tier zero: Cramer classification}

Applying the exclusionary method (see section "Category attribution (exclusionary method)") to the Cramer classification-derived probability distributions presented within Table 5 produced overly conservative estimations of acute toxicity category (presented in the form of a confusion matrix applicable to both 5\% and 10\% thresholdsTable 7). As is expressed within Fig. 4, 44 compounds from 50 were predicted to hold categories indicative of higher toxicity than that which they exhibit in vivo-that is, they are overpredicted. This is largely a consequence of the automatic assignment of Cramer class III compounds (46 from 50, including nine Category 4 and seven Category 5) to toxicity Category 1 . Given the extremity of the toxicity represented by such a label, it is inevitable that this relationship shall, in the great majority of instances, produce marked overstatement of associated hazard. The four compounds falling into Cramer class I were each attributed Category 3-again, a cautious assessment of their experimentally-defined toxicity.

\section{Tier one: in silico (EPA TEST + random forest + structural alerts)}

Through adoption of a Bayesian statistical approach (as outlined within section "Bayesian analysis"), Cramer classification-derived distributions were supplemented with outcomes from the aforementioned suite of in silico techniques: EPA TEST, random forest and structural alerts. Their effect, as anticipated, was to mitigate against the excessively conservative nature of the Tier 0 assignments—producing a 


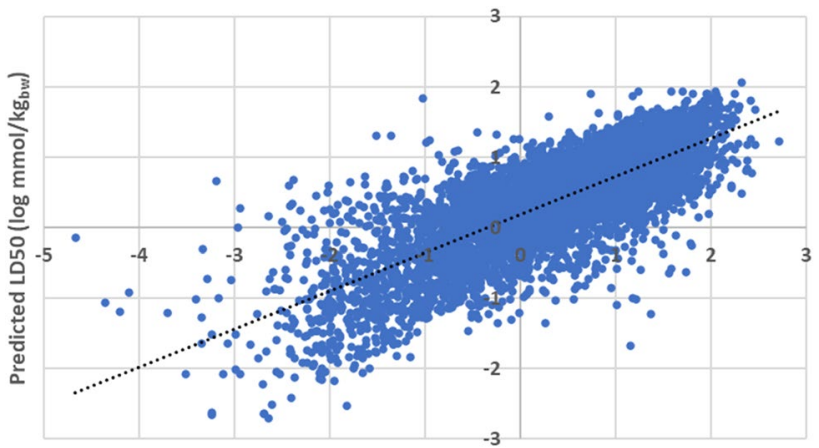

Experimental LD50 (log $\left.\mathrm{mmol} / \mathrm{kg}_{\mathrm{bw}}\right)$

Fig. 2 Plot outlining correlation between acute oral LD50 as predicted through random forest model, and that determined experimentally $\left(r^{2}=0.602\right)$

series of category-level predictions exhibiting a far greater degree of resolution and balance. Extent of overprediction was noted to fall, whilst instance of correct assignment grew (see matrices presented within Table 8).

Comparing within-tier, the inherent enhanced conservatism of the $5.0 \%$ exclusionary threshold relative to $10.0 \%$ was readily apparent. With application of the $5.0 \%$ threshold, quantity of compounds for which toxicity category

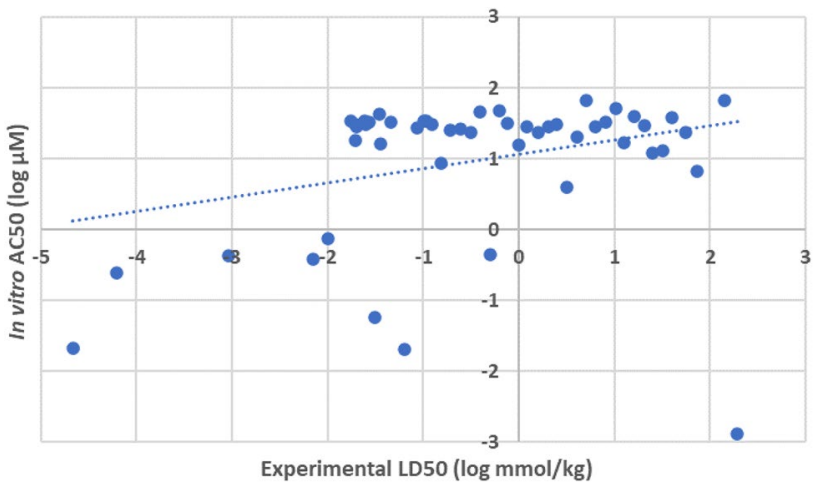

Fig. 3 Plot outlining correlation between in vitro cytotoxicity (expressed as $\log$ AC50) and acute oral LD50 $\left(r^{2}=0.0877\right)$

was predicted correctly rose from six (in Tier 0 ) to ten. On $10.0 \%$, this expanded further to fifteen. Concurrently, the number overpredicted fell from 44 to 39 and 33, respectively. Severity of this overprediction was notably diminishedwith the proportion of substances assigned categories three or four increments higher than their experimental equivalents (for example, Category 5 predicted either as Category 1 or 2) falling at the expense of those merely one or two higher. This may be seen reflected in the general shift from darker to lighter blue shades as evident within Fig. 4. With

Table 6 Overview of structural alerts present within ten or greater compounds

\begin{tabular}{|c|c|c|c|c|c|c|c|c|}
\hline \multirow[t]{3}{*}{ Alert title } & \multirow[t]{3}{*}{ Defining structure } & \multirow[t]{3}{*}{ Mechanistic considerations } & \multicolumn{5}{|c|}{ Scaled prob. distribution (\%) } & \multirow[t]{3}{*}{ Coverage } \\
\hline & & & \multicolumn{5}{|c|}{ Acute toxicity category } & \\
\hline & & & 1 & 2 & 3 & 4 & 5 & \\
\hline Organophosphate & {$[\mathrm{O}, \mathrm{S}]$} & $\begin{array}{l}\text { Neurotoxin } \\
\text { (acetylcholinesterase inhibition) }\end{array}$ & 41.8 & 33.9 & 16.7 & 4.6 & 3.1 & 730 \\
\hline Carbamate & & $\begin{array}{l}\text { Neurotoxin } \\
\text { (acetylcholinesterase inhibition) }\end{array}$ & 31.5 & 40.0 & 16.4 & 8.3 & 4.2 & 327 \\
\hline $\begin{array}{l}\text { Fluoromethyl- } \\
\text { benzimidazole } \\
\text { (fenazaflor-like) }\end{array}$ & & $\begin{array}{l}\text { Apparent inhibition of } \\
\text { oxidative phosphorylation }\end{array}$ & 51.6 & 41.3 & 7.0 & 0.0 & 0.0 & 128 \\
\hline $\begin{array}{l}\text { Vitamin K } \\
\text { antagonist } \\
\text { (warfarin-like) }\end{array}$ & & Anti-coagulant & 84.4 & 5.8 & 7.6 & 2.2 & 0.0 & 11 \\
\hline Dibenzodioxin & & Uncertain & 96.3 & 3.7 & 0.0 & 0.0 & 0.0 & 10 \\
\hline
\end{tabular}

Depicted is the defining structural fragment, alongside details relating to the known toxic mechanism associated with the class, its scaled category-probability distribution and its absolute coverage 
Table 7 Confusion matrix outlining category assignment following application of Tier 0 (applicable both to exclusionary thresholds $5.0 \%$ and $10.0 \%)$

\begin{tabular}{|c|c|c|c|c|c|c|}
\hline & & \multicolumn{5}{|c|}{ Category (predicted) } \\
\hline & & 1 & 2 & 3 & 4 & 5 \\
\hline \multirow[t]{5}{*}{ Category (experimental) } & 1 & 6 & 0 & 0 & 0 & 0 \\
\hline & 2 & 14 & 0 & 0 & 0 & 0 \\
\hline & 3 & 10 & 0 & 0 & 0 & 0 \\
\hline & 4 & 9 & 0 & 1 & 0 & 0 \\
\hline & 5 & 7 & 0 & 3 & 0 & 0 \\
\hline
\end{tabular}

adoption of the $10.0 \%$ threshold, 42 from the 50 compounds were attributed categories either identical to, or one step more conservative than, experimental. By contrast, instances of underprediction, absent entirely at Tier 0 , were observed. Quantities, however, were minimal-limited only to a single substance at the $5.0 \%$ limit, and to two at $10 \%$.

To further illustrate the general progression in prediction suitability, specific reference is made to performance against three compounds-each depicted within Fig. 5. These are the Category 3 substance 2,6-di-tert-butyl-4-nitrophenol (DTBNP; ID 4682), the Category 4 sodium bithionolate (ID 2365) and the Category 5 succinimide (ID 6524). Tier 0 assigns each to Category 1 -a clear overstatement of measured toxicity. Upon application of the Tier 1 model, figures are revised: 2,6-di-tert-butyl-4-nitrophenol to Categories 2 (5\% exclusionary threshold) or 3 (10\% exclusionary threshold), sodium bithionolate to Categories 3 or 4 respectively, and succinimide uniformly to Category 4 . A visual depiction of the variation in prediction accuracy is presented within Table 9 (being an excerpt from the broader "Predictivity heatmap" present within Supplementary Table 2).

\section{Tier two: in vitro cytotoxicity}

Further updating of the Bayesian model through incorporation of in vitro cytotoxicity outcomes served to have only minor impact upon performance, relative to that of Tier 1 . At the broadest level, an extremely modest shift away from tendency towards conservatism was witnessed-with the quantity of compounds overpredicted falling from 39 and 33 within Tier 1 (at the $5.0 \%$ and $10.0 \%$ thresholds respectively) to 37 and 32 . These drops were compensated directly by increases in the number of substances assigned to their correct categories, as extent of underprediction remained constant (refer to Table 10).

The described trends were reflected within each of the three exemplar compounds referenced previously, with variation from Tier 1 being either absent in its entirety or instead tending very mildly away from overprediction (Table 9). Category assignments of 2,6-di-tert-butyl-4-nitrophenol remained completely unchanged. Those of sodium bithionolate and succinimide were subject to minor alteration: the former now predicted as Category 4 (with application of either exclusion threshold), and the latter seeing its attribution at the $10.0 \%$ threshold switch from Category 4 to 5 (in line with experimentally-derived classification).
Fig. 4 Variation in extent of deviation in category assignment relative to experimentally determined classification, in accordance with assessment tier and exclusionary threshold. Colouration relates to extent of over- or underprediction, defined as differential between predicted and experimental toxicity categories

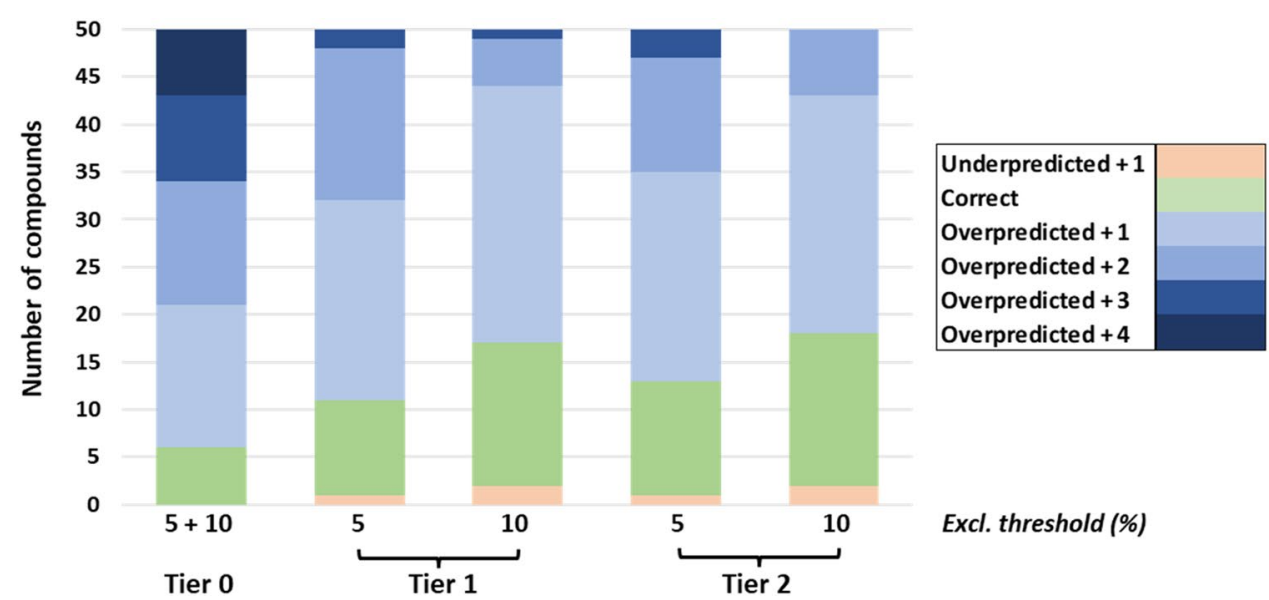


Table 8 Confusion matrices outlining category assignment following application of Tier 1 , following application of exclusionary thresholds $5.0 \%$ (a) and $10.0 \%$ (b)

\begin{tabular}{|c|c|c|c|c|c|c|}
\hline & & \multicolumn{5}{|c|}{ Category (predicted) } \\
\hline & & 1 & 2 & 3 & 4 & 5 \\
\hline \multirow{7}{*}{$\begin{array}{l}\text { (a) Category } \\
\text { (experimental) }\end{array}$} & 1 & 5 & 1 & 0 & 0 & 0 \\
\hline & 2 & 10 & 4 & 0 & 0 & 0 \\
\hline & 3 & 5 & 5 & 0 & 0 & 0 \\
\hline & 4 & 0 & 7 & 2 & 1 & 0 \\
\hline & 5 & 0 & 2 & 4 & 4 & 0 \\
\hline & & \multicolumn{5}{|c|}{ Category (predicted) } \\
\hline & & 1 & 2 & 3 & 4 & 5 \\
\hline \multirow{5}{*}{$\begin{array}{l}\text { (b) Category } \\
\text { (experimental) }\end{array}$} & 1 & 4 & 2 & 0 & 0 & 0 \\
\hline & 2 & 8 & 6 & 0 & 0 & 0 \\
\hline & 3 & 1 & 8 & 1 & 0 & 0 \\
\hline & 4 & 0 & 1 & 7 & 2 & 0 \\
\hline & 5 & 0 & 1 & 3 & 4 & 2 \\
\hline
\end{tabular}

\section{Discussion}

Concerns have been expressed over the reluctance of bodies such as regulatory agencies to adopt new approaches in the assessment of chemical safety for human health (Knight et al. 2021; Parish et al. 2020). A key consequence of this has been the continued employment of in vivo protocols, which has in turn, owing to limits imposed by financial cost and availability of expertise, restricted the volume of substances which may feasibly be screened. In many cases, these animal-based laboratory techniques were developed more than 50 years ago. The static nature of this situation indicates in general a lack of confidence in NAM-a position which we postulated may have arisen from inadequacies both in the techniques themselves, and also in the manner through which they have been validated (Mahony et al. 2020).

There was once an expectation that there would be direct one-to-one replacement of existing laboratory animal-based protocols with appropriate in vitro tests (Piersma et al. 2018). However, it has been demonstrated, even in such relatively simple endpoints as localised irritation, that it is beyond the capability of a single assay to cover the range of modes of action which may contribute to ultimate adverse effect (Piersma et al. 2014). The development of Integrated Approaches to Testing and Assessment (IATA) and tiered approaches has seen different sources of information, such as physicochemical properties, QSAR and in vitro methodologies, brought together to ascribe potential for toxicity (Worth and Patlewicz 2016). The outputs from methods combining different data sources exist in the form of probability distributions, not fixed points. Accordingly, they do not lend themselves well to specificity/sensitivity type analysis-in turn making it difficult for regulators to formulate and justify decisions in an objective fashion.

The purpose of this study has been to assess how, through integrating information sourced from various NAM (both in silico and in vitro) into a tiered assessment approach, it might prove possible to enhance overall confidence in the validity of conclusions over those drawn from individual techniques. Acute oral lethality was chosen as the endpoint of interest, owing to its intermediate complexity: whilst many modes of action may contribute, it lacks much of the additional ambiguity inherent within studies of repeatdose toxicity. Bayesian methodology was adopted as a means through which the information gathered from these approaches might be combined quantitatively, enabling reliable analysis of the contribution added by each to the overall verdict. This was itself expressed in terms of the probability that a substance might belong to one of five categories, each corresponding to defined ranges of LD50.

When considering a range of possibilities, it may be valuable to initially exclude those clearly least likely. Such an approach was adopted in the attribution of toxicity categories to compounds, producing an "exclusionary method". This proved to have an inherent conservatism, the extent of which could be adjusted through variation of the probability threshold beneath which a category was eliminated. This tendency to overstate hazard is, within limits, an acceptable feature for safety assessments. Within the study, chemicals were assigned to the most severe category holding probability greater than either 5.0 or $10.0 \%$-with the lower value naturally leading to the more conservative judgment. This protocol allowed for the tiered approach to be used with quantifiable certainty, enhancing confidence in decision 
<smiles>CC(C)(C)c1cc([N+](=O)[O-])cc(C(C)(C)C)c1O</smiles>
(DTBNP)<smiles>O=[N+]([O-])c1c(Cl)cc(Cl)cc1Sc1cc(Cl)cc(Cl)c1[O-]</smiles>

Sodium bithionolate

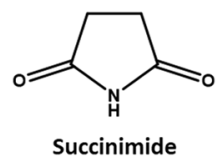

Fig. 5 Structures of each of the three illustrative compounds: DTBNP (experimental Category 3), sodium bithionolate (Category 4) and succinimide (Category 5)

making and in turn providing objective justification for its adoption. In principle, the methodology could be applied to other areas of toxicity for which a large reference database is present-for instance in determining repeat-dose derived no-effect levels (DNEL) from in vitro studies.

Analysis revealed that the introduction of a second tier of assessment (in silico) imparted substantial improvement in the balance of category assignments, relative to that obtained from the Cramer classification. This is to be anticipated, owing to the fact that Cramer's scheme was devised for the purposes of a conservative TTC assignment in which the great majority of compounds, with the exception of those most definitively recognised as inert, were assumed to exhibit a uniform toxicity (EFSA Scientific Committee 2019; Cramer et al. 1978). Both the EPA Test method (a series of linked QSARs), and the random forest algorithm (trained using a combination of molecular fingerprint fragments and physicochemical descriptors) contributed greatly to enhancing predictive resolution. These techniques generated estimates at the level of LD50, which could in turn input directly into the Bayesian model. It should be noted that there is potential overlap between the training data adopted in construction of TEST hierarchical clustering and that present within the Gadaleta et al. set. Since scope for improvement

existed within the performance of each $\left(r^{2}\right.$ values equal to 0.74 and 0.60 , respectively), the further addition of specified molecular structural alerts was considered worthwhile as a route towards addressing the issues arising from presence of outliers. As powerful as the pattern-recognition capacity of QSAR and machine learning might be, this potential can only be realised in the presence of adequate data (Sheridan 2012). As such, it is perhaps inevitable that high toxic potential within compounds possessing unique and complex structural motifs might evade detection. The creation of specific alerts relating families such as trichothecene, saxitoxin, aflatoxin was intended to mitigate this.

By contrast, the in vitro cytotoxicity data contributed little significant information when integrated within the tiered approach—producing only a very moderate redistribution in category assignment. Given the poor correlation between EC50 and LD50, this is very much to be expected. Whilst alternative studies have noted the improved performance of assays such as neutral red uptake (3T3 cells) in capturing in vivo lethality (Prieto Peraita et al. 2013), uncertainties remain concerning the general applicability of the methodology (Schrage et al. 2011). Effective or otherwise, scope for wider use is at present limited on account of the comparatively small quantities of compounds screened. In practice, we were restricted with respect to the data which could realistically be adopted: any candidate system would necessarily be required to cover a significant proportion of the compound inventory set. ToxCast proved the only readily available source matching this description-although the performance of the HEK-293 (human embryonic kidney) viability assay was clearly sub-optimal. This is a predicament which is highly likely to persist until the spread of substances tested through more suitable approaches, such as the aforementioned neutral red uptake screen, is systematically expanded. Shortcomings are even then likely still to remain, owing to the inherent challenges in interpolating organism lethality from the outcomes of simple, twodimensional cell-based in vitro techniques (Ekwall 1983; Garle et al. 1994). Modes of toxic action such as coagulation impairment and neurotoxicity are, amongst others, liable to be overlooked through such methods - and would perhaps be best accounted for during the in silico assessment phase.

Table 9 Variation in extent of deviation in category assignment relative to experimentally determined classification for three representative compounds: DTBNP (experimental Category 3), sodium bithionolate (Category 4) and succinimide (Category 5)

\begin{tabular}{|c|c|c|c|c|c|c|}
\hline Assessment tier & Tier 0 & \multicolumn{2}{|c|}{ Tier 1} & \multicolumn{2}{|c|}{ Tier 2} & \multirow{2}{*}{$\begin{array}{c}\text { Correct } \\
O P+1\end{array}$} \\
\hline Exclusionary threshold (\%) & $5+10$ & 5 & 10 & 5 & 10 & \\
\hline DTBNP & & & & & & $O P+2$ \\
\hline Sodium bithionolate & & & & & & $O P+3$ \\
\hline Succinimide & & & & & & $O P+4$ \\
\hline
\end{tabular}

Colouration relates to extent of overprediction $(\mathrm{OP})$, defined as differential between predicted and experimental toxicity categories 
Table 10 Confusion matrices outlining category assignment following application of Tier 2, following application of exclusionary thresholds $5.0 \%$ (a) and $10.0 \%$ (b)

\begin{tabular}{|c|c|c|c|c|c|c|}
\hline & & \multicolumn{5}{|c|}{ Category (predicted) } \\
\hline & & 1 & 2 & 3 & 4 & 5 \\
\hline \multirow{7}{*}{$\begin{array}{l}\text { (a) Category } \\
\text { (experimental) }\end{array}$} & 1 & 5 & 1 & 0 & 0 & 0 \\
\hline & 2 & 10 & 4 & 0 & 0 & 0 \\
\hline & 3 & 3 & 7 & 0 & 0 & 0 \\
\hline & 4 & 0 & 5 & 3 & 2 & 0 \\
\hline & 5 & 0 & 3 & 4 & 2 & 1 \\
\hline & & \multicolumn{5}{|c|}{ Category (predicted) } \\
\hline & & 1 & 2 & 3 & 4 & 5 \\
\hline \multirow{5}{*}{$\begin{array}{l}\text { (b) Category } \\
\text { (experimental) }\end{array}$} & 1 & 4 & 2 & 0 & 0 & 0 \\
\hline & 2 & 7 & 7 & 0 & 0 & 0 \\
\hline & 3 & 0 & 9 & 1 & 0 & 0 \\
\hline & 4 & 0 & 2 & 6 & 2 & 0 \\
\hline & 5 & 0 & 0 & 5 & 3 & 2 \\
\hline
\end{tabular}

As alluded to with section "Overview of tiered approach", a further tier (Tier 3), may be introduced to incorporate the targeted application of in vivo testing. This would be considered necessary only if there was large uncertainty with respect to the final verdict, or there existed an imperative to otherwise challenge the assigned category. This might arise for commercial reasons-for example, if an important product was to be attributed a category suggesting severe toxicity, in turn necessitating its exclusion. Alternatively, there may be concerns that a chemical had been predicted as low toxicity, despite the presence of additional evidence suggesting that it might in fact present a greater hazard. In these cases, it would not be necessary to carry out a full assessment of LD50. Instead, a refined means of assessing acute lethality, such as the OECD Guideline 425 (OECD 2008), could be employed. In brief, this is a stepwise procedure utilising single animals, whereby the first receives a dose marginally below the best estimate of the LD50 (which would itself be derived from Tier 2). Depending on the outcome for the previous animal, the dose for the next is increased or decreased-usually by a factor of 3:2. This sequence continues until there is a reversal of the initial outcome (i.e., the point where an increasing dose results in death rather than survival, or decreasing dose results in survival rather than death); at this stage, additional animals are dosed. The exclusionary method adopted within this study could be used to limit the use of further animals if no response is seen at a dose which would provide a level of confidence indicating that a particularly severe category of concern could safely be excluded.

Our investigation has indicated that Bayesian methodology can be used in the development and evaluation of tiered approaches for acute lethality assessment. Accordingly, the question arises of how successful such a technique might be if applied to alternative manifestations of toxicity. Previously, Bayesian analysis has been employed in the integration of data describing drug-induced liver injury (Semenova et al. 2020; Williams et al. 2020), cardiotoxicity (Felli and Leishman 2020) and skin sensitisation (Reynolds et al. 2019). Whilst potencies in many toxicological endpoints may be expressed readily within categorical terms (this format being particularly amenable to Bayesian treatment), there remain those for which such framing is not so practical (National Research Council 2014). Although it is comparatively trivial to assign bands of potency to points of departure, it is more difficult to imagine how ranges of effects could similarly be categorised. The idea of "protection not prediction" challenges whether the "free form" recording we currently have of adverse effects is necessary. Would it be sufficient simply to categorise outcomes as "severe", "moderate" or "mild" in order for risk assessment and risk management to operate? In more general terms, use of the tiered approach is dependent upon both the sourcing of large inventories containing outcomes of conventional laboratory animal-based protocols, and on the acquisition of NAM data (in the form of relevant in vitro endpoints, QSAR etc.) which may readily be integrated across the various stages of assessment. Some difficulties were experienced in accessing data on account of the varying formats within which it may be held. Agreement on the formatting of databases containing in vivo and in vitro outcomes would enable the development of methodology in which regulatory authorities could have confidence.

In conclusion, we have shown that Bayesian inference can be adopted to integrate data from a variety of NAM 
sources-producing output from in the form of probability distributions which may subsequently be used in provision of objective assessments of toxic potential (in this instance, acute oral lethality). An exclusionary approach enabled assignment of chemicals to established toxicity categories, with a defined level of certainty. In terms of validation, this has potential to offer increased confidence relative to the simple quantification of sensitivity and specificity. Whilst the remit of this particular investigation has remained limited to the relatively narrow area of acute lethality, there is great scope for wider application of the approach. Accordingly, it is our intention that the demonstrated methodology might ultimately serve in providing a level of confidence in NAM sufficient to gain wider use in regulatory decision making-offering as it does the benefit of reducing the number of laboratory animals used, in turn allowing more efficient assessment of chemicals within existing financial and expert resources.

Supplementary Information The online version contains supplementary material available at https://doi.org/10.1007/s00204-021-03205-x.

Acknowledgements We are grateful to Amelia Doe for her helpful suggestions in developing the exclusionary method for assignment of chemicals to categories.

Funding No funding was received for conducting this study.

Availability of data and materials The authors confirm that the data supporting the findings of this study are available within the article and its supplementary materials.

Code availability Not applicable.

\section{Declarations}

Conflict of interest The authors have no conflicts of interest to declare that are relevant to the content of this article.

Open Access This article is licensed under a Creative Commons Attribution 4.0 International License, which permits use, sharing, adaptation, distribution and reproduction in any medium or format, as long as you give appropriate credit to the original author(s) and the source, provide a link to the Creative Commons licence, and indicate if changes were made. The images or other third party material in this article are included in the article's Creative Commons licence, unless indicated otherwise in a credit line to the material. If material is not included in the article's Creative Commons licence and your intended use is not permitted by statutory regulation or exceeds the permitted use, you will need to obtain permission directly from the copyright holder. To view a copy of this licence, visit http://creativecommons.org/licenses/by/4.0/.

\section{References}

Anadón A, Martínez MA, Castellano V, Martínez-Larrañaga MR (2014) The role of in vitro methods as alternatives to animals in toxicity testing. Expert Opin Drug Metab Toxicol 10(1):67-79. https://doi.org/10.1517/17425255.2014.854329

Andersen ME, McMullen PD, Phillips MB et al (2019) Developing context appropriate toxicity testing approaches using new alternative methods (NAMs). Altex 36(4):523-534. https://doi.org/10. 14573/altex.1906261

Berthold MR, Cebron N, Dill F et al (2008) KNIME: the Konstanz information miner. In: Preisach C, Burkhardt H, Schmidt-Thieme L, Decker R (eds) Data analysis, machine learning and applications. Springer, Berlin, Heidelberg, pp 319-326

Carpenter B, Gelman A, Hoffman MD et al (2017) Stan: a probabilistic programming language. J Stat Softw 76(1):32. https://doi.org/10. 18637/jss.v076.i01

Clothier R, Dierickx P, Lakhanisky T et al (2008) A database of IC50 values and principal component analysis of results from six basal cytotoxicity assays, for use in the modelling of the in vivo and in vitro data of the EU ACuteTox project. Altern Lab Anim 36(5):503-519. https://doi.org/10.1177/026119290803600509

Cramer GM, Ford RA, Hall RL (1978) Estimation of toxic hazarda decision tree approach. Food Cosmet Toxicol 16(3):255-276. https://doi.org/10.1016/s0015-6264(76)80522-6

Dal Negro G, Eskes C, Belz S et al (2018) One science-driven approach for the regulatory implementation of alternative methods: a multisector perspective. Regul Toxicol Pharmacol 99:33-49. https:// doi.org/10.1016/j.yrtph.2018.08.002

Edwards SW, Tan Y-M, Villeneuve DL, Meek ME, McQueen CA (2016) Adverse outcome pathways - organizing toxicological information to improve decision making. J Pharmacol Exp Ther 356(1):170. https://doi.org/10.1124/jpet.115.228239

Ekwall B (1983) Correlation between cytotoxicity in vitro and LD50values. Acta Pharmacol Toxicol 52(s2):80-99. https://doi.org/10. 1111/j.1600-0773.1983.tb02685.x

European Union (2008) Regulation (EC) No 1272/2008 of the European Parliament and of the Council of 16 December 2008 on classification, labelling and packaging of substances and mixtures, amending and repealing Directives 67/548/EEC and 1999/45/ EC, and amending Regulation (EC) No 1907/2006. https://eurlex.europa.eu/legal-content/EN/TXT/?uri=OJ:L:2008:353:TOC

Felli JC, Leishman DJ (2020) A Bayesian approach to toxicological testing. J Pharmacol Toxicol Methods 105:106898. https://doi. org/10.1016/j.vasen.2020.106898

Gadaleta D, Vuković K, Toma C et al (2019) SAR and QSAR modeling of a large collection of LD50 rat acute oral toxicity data. J Cheminform 11(1):58. https://doi.org/10.1186/s13321-019-0383-2

Garle MJ, Fentem JH, Fry JR (1994) In vitro cytotoxicity tests for the prediction of acute toxicity in vivo. Toxicol Vitro 8(6):1303-1312. https://doi.org/10.1016/0887-2333(94)90123-6

Kinsner-Ovaskainen A, Prieto P, Stanzel S, Kopp-Schneider A (2013) Selection of test methods to be included in a testing strategy to predict acute oral toxicity: an approach based on statistical analysis of data collected in phase 1 of the ACuteTox project. Toxicol Vitro 27(4):1377-1394. https://doi.org/10.1016/j.tiv.2012.11.010

Kleinstreuer NC, Karmaus A, Mansouri K, Allen DG, Fitzpatrick JM, Patlewicz G (2018) Predictive models for acute oral systemic toxicity: a workshop to bridge the gap from research to regulation. Comput Toxicol 8(11):21-24. https://doi.org/10.1016/j.comtox. 2018.08.002

Knight DJ, Deluyker H, Chaudhry Q, Vidal J-M, de Boer A (2021) A call for action on the development and implementation of new methodologies for safety assessment of chemical-based products in the EU-a short communication. Regul Toxicol Pharmacol 119:104837. https://doi.org/10.1016/j.yrtph.2020.104837

Krewski D, Acosta D, Andersen M et al (2010) Toxicity testing in the 21st century: a vision and a strategy. J Toxicol Env Health Part B 13(2-4):51-138. https://doi.org/10.1080/10937404.2010.483176 Landrum G (2006) RDKit: open-source cheminformatics 
Lazic SE, Williams DP (2021) Quantifying sources of uncertainty in drug discovery predictions with probabilistic models. Artif Intell Life Sci 1:100004. https://doi.org/10.1016/j.ailsci.2021.100004

Liaw A, Wiener M (2002) Classification and regression by randomForest. R News 2(3):18-22

Madden JC, Enoch SJ, Paini A, Cronin MTD (2020) A review of in silico tools as alternatives to animal testing: principles, resources and applications. Altern Lab Anim 48(4):146-172. https://doi.org/ 10.1177/0261192920965977

Mahony C, Ashton RS, Birk B et al (2020) New ideas for non-animal approaches to predict repeated-dose systemic toxicity: report from an EPAA Blue Sky Workshop. Regul Toxicol Pharmacol 114:104668. https://doi.org/10.1016/j.yrtph.2020.104668

Martin TM, Harten P, Venkatapathy R, Das S, Young DM (2008) A hierarchical clustering methodology for the estimation of toxicity. Toxicol Mech Methods 18(2-3):251-266. https://doi.org/10.1080/ 15376510701857353

Molecular Operating Environment (2018) Chemical computing group ULC. https://www.chemcomp.com/

National Research Council (2014) Appendix D: overview of the GHS classification scheme in hazard classification a framework to guide selection of chemical alternatives. National Academies Press (US), Washington (DC). https://doi.org/10.17226/18872

Nelms MD, Karmaus AL, Patlewicz G (2020) An evaluation of the performance of selected (Q)SARs/expert systems for predicting acute oral toxicity. Comput Toxicol 16:100135. https://doi.org/10. 1016/j.comtox.2020.100135

OECD (2008) Test No. 425: acute oral toxicity: up-and-down procedure. OECD guidelines for the testing of chemicals, section 4. OECD Publishing, Paris. https://doi.org/10.1787/9789264071 049-en

Parish ST, Aschner M, Casey W et al (2020) An evaluation framework for new approach methodologies (NAMs) for human health safety assessment. Regul Toxicol Pharmacol 112:104592. https://doi.org/ 10.1016/j.yrtph.2020.104592

Patlewicz G, Jeliazkova N, Safford RJ, Worth AP, Aleksiev B (2008) An evaluation of the implementation of the Cramer classification scheme in the Toxtree software. SAR QSAR Environ Res 19(5-6):495-524. https://doi.org/10.1080/10629360802083871

Piersma AH, Ezendam J, Luijten M et al (2014) A critical appraisal of the process of regulatory implementation of novel in vivo and in vitro methods for chemical hazard and risk assessment. Crit Rev Toxicol 44(10):876-894. https://doi.org/10.3109/10408444. 2014.940445

Piersma AH, Burgdorf T, Louekari K et al (2018) Workshop on acceleration of the validation and regulatory acceptance of alternative methods and implementation of testing strategies. Toxicol Vitro 50:62-74. https://doi.org/10.1016/j.tiv.2018.02.018

Prieto P, Kinsner-Ovaskainen A, Stanzel S et al (2013) The value of selected in vitro and in silico methods to predict acute oral toxicity in a regulatory context: results from the European Project ACuteTox. Toxicol Vitro 27(4):1357-1376. https://doi.org/10. 1016/j.tiv.2012.07.013

Prieto Peraita M, Griesinger C, Amcoff S, Whelan M (2013) EURL ECVAM Recommendation on the 3T3 neutral red uptake cytotoxicity assay for acute oral toxicity testing. Publ Office Eur Union. https://doi.org/10.2788/88799

R Core Team (2021) R: a language and environment for statistical computing. In: R Foundation for Statistical Computing. https:// www.R-project.org/

Reynolds J, MacKay C, Gilmour N, Miguel-Vilumbrales D, Maxwell G (2019) Probabilistic prediction of human skin sensitiser potency for use in next generation risk assessment. Comput Toxicol 9:3649. https://doi.org/10.1016/j.comtox.2018.10.004

Richard AM, Judson RS, Houck KA et al (2016) ToxCast chemical landscape: paving the road to 21 st century toxicology. Chem Res
Toxicol 29(8):1225-1251. https://doi.org/10.1021/acs.chemrestox. $6 \mathrm{~b} 00135$

RStudio Team (2020) RStudio: integrated development for R. In: RStudio, PBC. http://www.rstudio.com/

Schrage A, Hempel K, Schulz M, Kolle SN, van Ravenzwaay B, Landsiedel R (2011) Refinement and reduction of acute oral toxicity testing: a critical review of the use of cytotoxicity data. Altern Lab Anim 39(3):273-295. https://doi.org/10.1177/0261192911 03900311

Scientific Committee EFSA, More SJ, Bampidis V et al (2019) Guidance on the use of the threshold of toxicological concern approach in food safety assessment. EFSA J 17(6):e05708. https://doi.org/ 10.2903/j.efsa.2019.5708

Semenova E, Williams DP, Afzal AM, Lazic SE (2020) A Bayesian neural network for toxicity prediction. Comput Toxicol 16:100133. https://doi.org/10.1016/j.comtox.2020.100133

Sheridan RP (2012) Three useful dimensions for domain applicability in QSAR models using random forest. J Chem Inf Model 52(3):814-823. https://doi.org/10.1021/ci300004n

Stan Development Team (2020) RStan: the R interface to Stan. http:// mc-stan.org/

Thomas RS, Philbert MA, Auerbach SS et al (2013) Incorporating new technologies into toxicity testing and risk assessment: moving from 21 st century vision to a data-driven framework. Toxicol Sci 136(1):4-18. https://doi.org/10.1093/toxsci/kft178

Thomas RS, Bahadori T, Buckley TJ et al (2019) The next generation blueprint of computational toxicology at the US environmental protection agency. Toxicol Sci 169(2):317-332. https://doi.org/ 10.1093/toxsci/kfz058

Tice RR, Austin CP, Kavlock RJ, Bucher JR (2013) Improving the human hazard characterization of chemicals: a Tox21 update. Environ Health Perspect 121(7):756-765. https://doi.org/10.1289/ ehp. 1205784

Tollefsen KE, Scholz S, Cronin MT et al (2014) Applying adverse outcome pathways (AOPs) to support integrated approaches to testing and assessment (IATA). Regul Toxicol Pharmacol 70(3):629-640. https://doi.org/10.1016/j.yrtph.2014.09.009

Vinken M (2013) The adverse outcome pathway concept: a pragmatic tool in toxicology. Toxicology 312:158-165. https://doi.org/10. 1016/j.tox.2013.08.011

Weininger D (1988) SMILES, a chemical language and information system. 1. Introduction to methodology and encoding rules. J Chem Inf Comput Sci 28(1):31-36. https://doi.org/10.1021/ci000 $57 \mathrm{a} 005$

Williams AJ, Grulke CM, Edwards J et al (2017) The CompTox chemistry dashboard: a community data resource for environmental chemistry. J Cheminform 9(1):61. https://doi.org/10.1186/ s13321-017-0247-6

Williams DP, Lazic SE, Foster AJ, Semenova E, Morgan P (2020) Predicting drug-induced liver injury with Bayesian machine learning. Chem Res Toxicol 33(1):239-248. https://doi.org/10.1021/ acs.chemrestox.9b00264

Worth AP, Patlewicz G (2016) Integrated approaches to testing and assessment. In: Eskes C, Whelan M (eds) Validation of alternative methods for toxicity testing. Springer International Publishing, Cham, pp 317-342

Publisher's Note Springer Nature remains neutral with regard to jurisdictional claims in published maps and institutional affiliations. 